Castañeda Arredondo, Erika Cecilia.

Alumna, Universidad Nacional Autónoma de México, Facultad de Artes y Diseño, Doctorado en Artes y Diseño.

\title{
Transborder Immigrant Tool. Lo real frente a lo virtual de una propuesta artística de movilidad en la frontera México - EE.UU.
}

TIPO DE TRABAJO

Comunicación

PALABRAS CLAVE

Lo real, virtual, movilidad, arte de frontera.

KEY WORDS

Real, virtual, mobility, art in the border.

\section{RESUMEN}

En esta comunicación abordaré la forma en que la diada real-virtual tiene una aplicación artística en el proyecto Transborder Immigrant Tool del colectivo de San Diego California-EEUU, Electronic Disturbance Theater. Este proyecto consiste en una aplicación GPS (Global Positioning System) para teléfono móvil que busca auxiliar a los migrantes en el desierto del Anza-Borrego, frontera EEUU - México. Los ayuda a localizar depósitos de agua, que han sido instalados por diferentes ONG de ayuda humanitaria, mediante una interfaz que les indica, a manera de una brújula, el camino a seguir, y además cuenta con una serie de poemas-instrucciones los cuales contienen datos útiles que ayudan a la supervivencia en el desierto.

Propongo considerar la diada real-virtual más que como una oposición, como una yuxtaposición entre lo real y lo posible por dos vías. La primera se refiere a la forma en que este proyecto cuestiona estéticamente la visibilidad del migrante en La Frontera, lugar que en su calidad de operador de lo real, excluye y moviliza al migrante como un dato, sujeto al control, al contraflujo y la vigilancia. La segunda busca explicar los niveles en los que opera la discusión acerca de lo posible en la obra de arte. Así en el nivel político permite reflexionar lo que puede ser posible, en el nivel tecnológico cómo hacerlo posible: el deseo; y el nivel estético nos hace volver sobre la imposibilidad del libre tránsito en la frontera, para oponer un imaginario de acceso y hospitalidad.

\section{ABSTRACT}

In this paper I will discuss how real-virtual dyad has an artistic application in the Transborder Immigrant Tool project from the San Diego California-USA collective, Electronic Disturbance Theater. This project consists of a GPS mobile phone application that seeks to assist migrants in the Anza-Borrego desert USA - Mexico border. It assists to locate water reservoirs which have been installed by several humanitarian NGO's, using a compass interface that indicates the way forward. The app has a series of poems-instructions which contain useful data to help survival into the desert.

I propose to consider real-virtual dyad rather than an opposition as a juxtaposition between what is real and what is possible in two ways. The first concerns the manner in which this project aesthetically interrogates the visibility of migrants in the border that is an operator of the real which functions through the mobilization and exclusion of the migrant as a subject to control and backflow monitoring. The second seeks to explain the levels at which the discussion about the possible operates in the artwork. So at the political level we can reflect what may be possible; at the technological level how to do it possible: the desire; and at the aesthetic level we turn back on the impossibility of free transit at the border, to set off an imaginary of access and hospitality. 
Comenzaré señalando un aspecto fundamental de Transborder Immigrant Tool (TBT): éste no ha podido ser implementado, aunque se encuentra en proceso de serlo a través de La Casa del Migrante en Tijuana - Baja California, México. ${ }^{1}$ La razón por la que no puede ser implementado en EEUU, responde a que los desarrolladores del mismo han sido sometidos a averiguaciones de orden judicial en este país durante 2010, por su supuesta ayuda en el cruce de inmigrantes ilegales y por malversación de fondos en la universidad de San Diego California (UCSD) que lo financió como un proyecto académico.
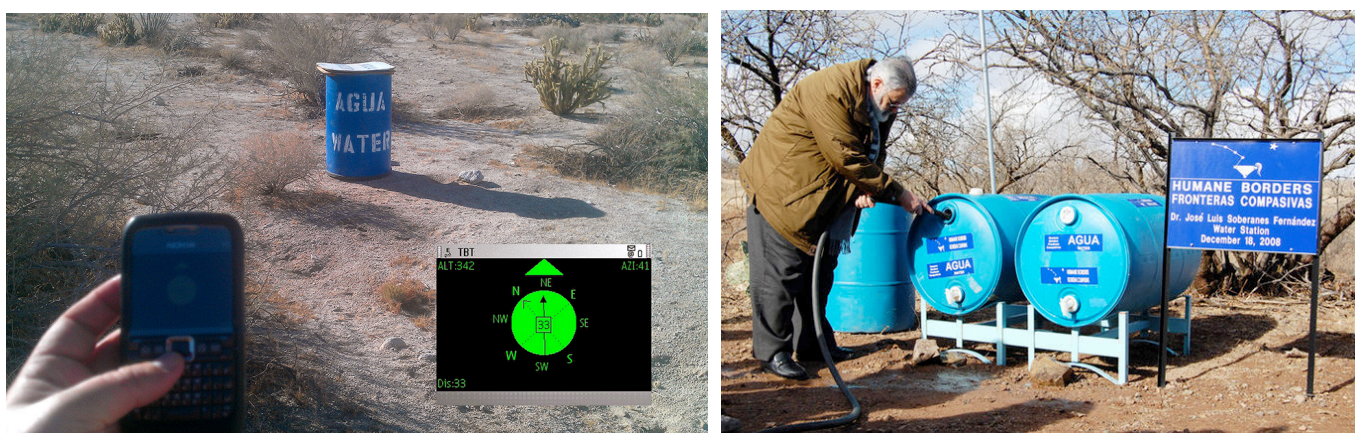

llustraciones 1 y 2 . Aplicación en uso y estaciones de agua por Humane Borders. Además de su operatividad como brújula y guía de orientación en el desierto, la aplicación cuenta con una serie de videos y poemas que contienen datos útiles e instrucciones para la supervivencia, como formas de localizar agua contenida en ciertos tipos de cactus, maneras de ubicar la estrella del norte (polaris), identificación de tipos de fauna nociva, etc.
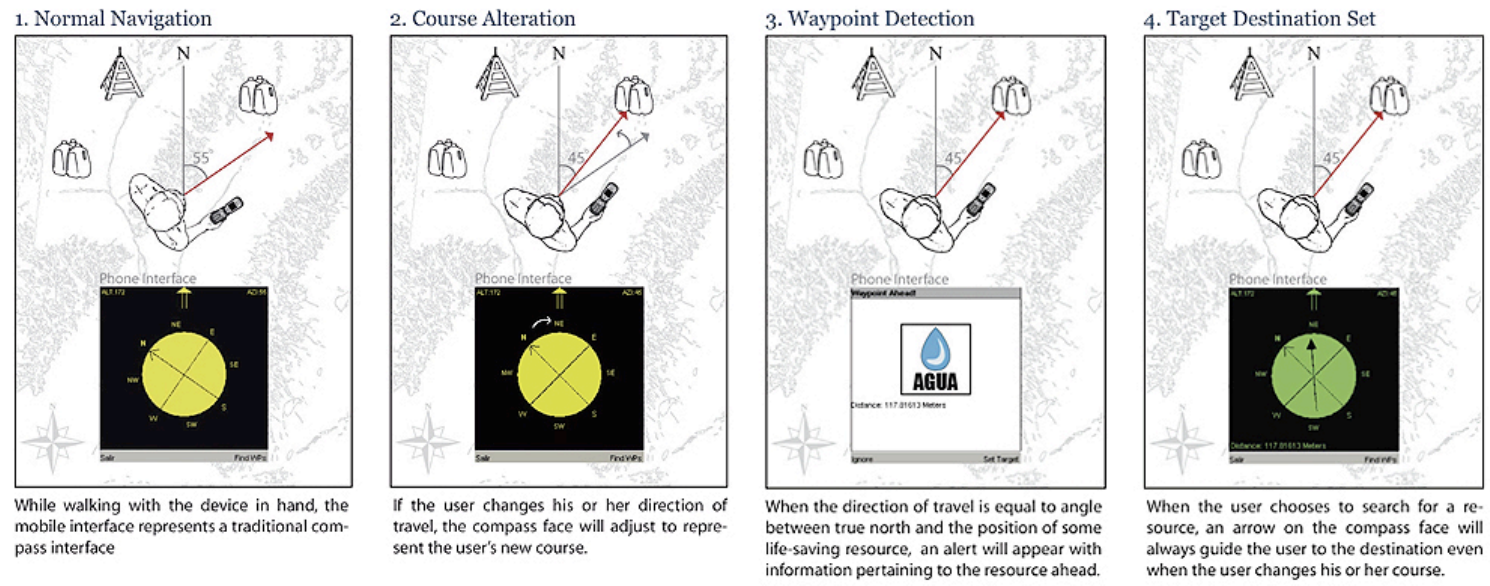

llustración 3. Descripción de uso de la app

Esta situación mantiene al proyecto en una suerte de estado de suspensión, esto es, al no poder ser utilizado por los migrantes, para quienes está destinado, ha suscitado otros tipos de gestiones, discusiones diversas y ha sido motivo de múltiples análisis en variadas direcciones. Aquí intentaré primero demostrar que, a pesar de estar en suspensión, es capaz de evidenciar las condiciones biopolíticas de la migración en la frontera México - EEUU², que es actualmente la frontera con mayor flujo migratorio en el mundo, lugar que funge como un operador de lo real, cuestión que argumentaré puntualmente en el primer apartado; y segundo, en discurrir sobre los modos en que la obra pone de manifiesto lo posible en una obra de arte contemporáneo, argumento sobre el que se concentrará el segundo apartado.

Expondré entonces, a modo de introducción, la problematización sobre los conceptos de lo real y lo virtual, en los que se basarán los argumentos de las dos partes que componen esta comunicación.

\footnotetext{
${ }^{1}$ Al respecto de esa intención, puede consultarse la conferencia dictada por Micha Cardenas, integrante del Electronic Disturbance Theater, autores del proyecto, en: http://bang.transreal.org/micha-cardenas-at-antiatlas/, consultada en noviembre de 2014. TBT constituye el tema de mi investigación como estudiante del Doctorado en Artes y Diseño de la FAD-UNAM, México.

${ }^{2}$ Por biopolítica entiendo técnicas de poder no disciplinario que se aplican a la vida de los hombres, al ser humano/ser viviente, como indica Michel Foucault, tecnologías que se concentran en procesos propios de la vida, como el nacimiento, la muerte, la enfermedad, la producción, etc., procesos que son objetos de saber y objetivos de control. Foucault, Michel: Defender la Sociedad, pp.220-221
} 
Pierre Lévy, señala que la realidad implica una actualización material, una presencia tangible, mientras lo virtual "procede del latín virtualis que deriva de virtus: fuerza - potencia. Existe en potencia pero no en acto, tiende a actualizarse, pero no se concretiza de modo efectivo o formal." ${ }^{3}$ Esta definición indica que estos conceptos pueden ser entendidos como yuxtapuestos y no como opuestos ni excluyentes. Así, para Gilles Deleuze en Diferencia y Repetición, lo posible, lo que existe en potencia, es un real fantasmagórico, latente, idéntico a lo real, sólo le falta la existencia, de tal suerte que la diferencia entre lo real y lo posible es puramente lógica. G. Deleuze también indica que lo real y lo posible están enlazados por la asociación entre el principio de realidad y el principio de placer, esto es, que la actividad real está determinada por una serie de excitaciones vinculadas con una intencionalidad, con algo por realizarse. Ese algo es un objeto virtual, un objeto transicional porque reclama un proceso de resolución. ${ }^{4}$ Me interesa particularmente hacer notar que este enlace, señala, entonces, los modos en que los objetos virtuales son incorporados a los objetos reales, incorporados más no integrados, esto es, se proyectan sobre los objetos reales que están regidos por el principio de realidad. El objeto virtual carece de algo en sí mismo, es la mitad de algo, su otra mitad está siempre ausente, la carta robada de Edgar Allan Poe que utiliza Jaques Lacan como ejemplo, "Lacan muestra que los objetos reales, en virtud del principio de realidad, están sometidos a la ley de estar $o$ de no estar en algún lugar, pero que el objeto virtual, por el contrario, tiene la propiedad de estar y de no estar, allí donde está, dondequiera que vaya...."

La yuxtaposición de estos dos conceptos, lo real y lo virtual como lo posible, conmina a preguntar en referencia a Transborder Immigrant Tool: ¿cuál es la condición real - virtual en el desierto fronterizo de EEUU en términos del tránsito migratorio ilegal, de qué manera este proyecto puede hacer visible esa condición, si no se encuentra en uso?, y ¿cómo se proyecta sobre esa realidad, lo posible de una poética como la de TBT?

\section{VISIBILIDAD DEL MIGRANTE EN LA FRONTERA. EL OPERADOR DE LA VIRTUALIZACIÓN.}

Sobre la base del entendimiento acerca de lo real y lo virtual como una yuxtaposición que está atravesada por el deseo de realización, comenzaré por determinar la configuración de la frontera, porque esencialmente este proyecto hace referencia por un lado al paisaje, lo agreste del desierto y sus condiciones específicas para la supervivencia; y por el otro, apunta su crítica hacia las condiciones biopolíticas del lugar, esto es, al constructo que implica el espacio frontera. De tal forma que tanto por sus condiciones medioambientales, como por su construcción conceptual, la frontera es una matriz: ordenación de elementos, conjunto de variables, conjunto de materiales que forman parte de un tejido, estructura en la que se insertan elementos operativos, en ese sentido es una actualización material, un arreglo de lo real. Esta matriz es una de orden instituyente, como institución regula y opera una cierta cantidad de elementos que caracterizan a una determinada espacialidad. ${ }^{6} \mathrm{Y}$ es además, un efecto del lenguaje que produce una dimensión lingüística, decir "Frontera" implica una realidad lingüística que opera instituyendo ese lugar como reconocimiento de un espacio real para la comunicación, el control, la vigilancia y el tránsito; es en otros términos, el efecto de la convención institucional, que busca regular, concentrar y limitar la movilidad de los cuerpos.

Así, podemos decir, que la frontera es una realidad actualizable que se performa, que se actúa, que tiene efectos activos en los cuerpos, por lo que es una matriz de tipo performativa. Hago aquí, con el fin de aclarar el concepto de performatividad, una analogía con la precisión sobre el mismo que apunta Bifo: "Lo que [John] Austin llama performatividad es un efecto que el lenguaje produce en una dimensión que es puramente lingüística, porque si yo digo 'los declaro marido y mujer' nada pasa realmente pero todo pasa en la dimensión de realidad lingüística. Es lenguaje como institución." "7 Me valgo también del uso del concepto que hace Judith Butler en su famoso ensayo El Género en Disputa. El feminismo y la subversión de la identidad (2007), en el cual retoma la discusión entre J. Austin y J. Derrida respecto del enunciado performativo, que ambos señalan como aquel que produce o transforma una situación: opera; pero a cuya descripción J. Butler añade, que éste tiene una historia, una significación sedimentada, y en ese sentido, ha contribuido a la construcción de enunciados legitimados y consolidados; y además J. Butler advierte sobre la dimensión dual de la performatividad: actúa sobre nosotros y nosotros actuamos, somos vulnerables y afectados por los discursos que no aceptamos, de tal suerte que las posibilidades de resistirlos, tienen dimensiones psíquicas, deseamos que las cosas no sean así, y dimensiones políticas, movilizamos nuestra vulnerabilidad, estamos expuestos y somos agentivos de esos discursos al mismo tiempo. ${ }^{8}$

La performatividad es, como se puede inferir, una condición de lo real, primero porque produce y opera, y luego porque su producción implica también, a un sujeto inteligible $o$ a un ininteligible, como señalaba J. Lacan apunta a la condición de estar $o$ no estar, si proyectamos esta condición a la realidad de la frontera, nos topamos de frente con la yuxtaposición real/virtual, la frontera se

\footnotetext{
${ }^{3}$ Lévy, Pierre: ¿Qué es lo virtual?, Ediciones Paidós, Barcelona, 1999, p.10

${ }^{4}$ Deleuze, Gilles: Diferencia y Repetición, Amorrortu Editores, Buenos Aires, 2002, pp.158 - 160

${ }^{5}$ Ibídem, p.162

${ }^{6}$ La frontera es un espacio de regulaciones y de divisiones, segmentaciones, racionalizaciones y racializaciones, y en ese sentido, es también lo que señala Henri Lefebvre en La Producción del Espacio: un tipo de espacio "científico", que es representacional del capitalismo, en el que el conocimiento está integrado y es integrador de las fuerzas de producción y de las relaciones sociales de producción; que implica una ideología diseñada para ocultar su uso... (p.9)

Berardi, Franco (Bifo): "The dark side of the multitud", en Colectivo Situaciones (Coord.), Impasse: dilemas politicos del presente, Buenos Aires: Tinta Limón, 2009, p.86

${ }^{8}$ Butler, Judith: Vulnerabilidad y Resistencia revisitadas, Conferencia, Sala Netzahualcóyotl, Ciudad Universitaria, UNAM, México D.F., 23 de marzo de 2015.
} 
caracteriza por producir a un sujeto/contexto de la migración: el migrante, que es su otra mitad, el objeto virtual cuya ontología estriba entre ser y no ser un sujeto de códigos, que eventualmente llegan a determinar la viabilidad de su vida.

Hacer visibles a los migrantes en el desierto fronterizo es cuestión operativa y cuestión vital, pero también es cuestión epistemológica dado que el migrante como categoría simbólica, nos permite problematizar la matriz, cuestionar su condición de lo real, para resistirla con lo posible y su imaginario.

\section{LOS NIVELES DE LO POSIBLE Y EL REGRESO A LA IMPOSIBILIDAD.}

Este proceso al que está expuesto el migrante, al que es vulnerable (el cruce del desierto es cuestión de vida o muerte), indica que la frontera se caracteriza además por una compleja virtualización de esa espacialidad, esto es, contrario a lo que podríamos pensar sobre el espacio frontera siguiendo el argumento de Hannah Arendt, como un espacio de aparición para una performatividad (romántica), la frontera es un espacio en el que se aparece $\boldsymbol{y}$ se desaparece ; en ella tiene lugar un proceso de virtualización que consiste en "un desplazamiento del centro de gravedad ontológico del objeto considerado [el migrante ilegal], que en lugar de definirse por su actualidad (una 'solución'), encuentra su consistencia esencial en un campo problemático"10, lo cual se acusa por las acciones del migrante calificadas como ilegales (fuera de la ley). Sus movimientos dependen de la espacialidad, dependen, aún antes que del uso de un GPS (Global Positioning System), de un conjunto de tecnologías que generalmente le son hostiles ${ }^{11}$, y toda esa hostilidad le confiere una condición de cuerpo vulnerable. Lo que busca generar en ese sentido TBT, a través del uso de una brújula que asiste en la localización de agua, es un cuerpo agencial, un cuerpo sostenido, cuya movilización supone un apoyo, y esto lo hace en lo posible, en la potencia de ser un acto que demanda una resolución. Resolución que además regresará al cuerpo, porque éste, como indica J. Butler, no está definido por sus límites sino por las relaciones que hacen posibles las vidas y sus posibles relaciones, posibles también por su interminable deseo del Norte. ${ }^{12}$

TBT, al apelar a esa condición, apunta no sólo a una espacialidad que es limítrofe y que está determinada por una operatividad de continua expulsión, sino que también nos permite ver que más que un límite, se trata de un limen, esta virtualización de la frontera apunta hacia una condición de liminalidad porque es indicio de aquello que no puede ser localizado en una estructura social, el migrante, espectro que ha partido y al cual no le ha sido dado llegar. ${ }^{13}$ Las condiciones del desierto agudizan la viabilidad de su vida, y a su vez, la condición performativa de la frontera, sus regulaciones y restricciones, ocluyen, desidentifican su presencia al oponer el velo de la duda sobre si éste vive o muere. Lo que TBT hace al pretender ponerlo en marcha sobre la ruta de su supervivencia a través del encuentro con las estaciones de agua, es como la pregunta del idiota, ¿vives o mueres?, o mejor aún ¿quieres vivir o quieres morir?, al hacer eso nos señala primero que en efecto, existen agentes cuya ontología en el presente parece indefinida, lo que esconde una suerte de vidas desechables, y a lo cual sólo podremos oponernos y resistir, si comenzamos por comprender que la virtualidad de la frontera radica en un debate por la viabilidad de la vida; y luego nos conmina a desestabilizar esa configuración de una forma particular, del orden de la representación en la que el migrante encuentra la ruta de su supervivencia y el acceso al Norte.

\footnotetext{
${ }^{9}$ La famosa distinción que hace Hannah Arendt entre vita activa y vita contemplativa, se funda en una jerarquía ontológica en la que la primera pertenece al orden de lo real y la segunda al orden de lo aparente, en donde la existencia es pasajera y aleatoria y los seres aparecen y desaparecen sin principio y sin finalidad. Contra esa división, Arendt propone un "espacio de aparición" cuyos rasgos distintivos son la pluralidad y la natalidad; la primera reúne y separa simultáneamente a diversos seres singulares y sus diversas percepciones del mundo, y la segunda implica que cada vida singular se inicia dotada de una agencia para comenzar algo nuevo en el mundo. Campillo, Antonio: "Espacios de aparición: el concepto de lo político en Hannah Arendt" en Daimon, No 26, 2002, España, pp.159186.

Digo que se aparece y se desaparece como un juego de conceptos, dado que lo que en términos arendtianos, aparece innegablemente es un corpus legal, que regula, una normatividad que define y brazos ejecutores de esa normatividad como la border patrol, añadamos el papel de grupos de vigilancia civil como los Minutemen. Lo que desaparece son seres humanos cuyas vidas están en suspenso, son literalmente desaparecidos, acerca de la mayoría de estos no sabemos si viven o mueren.

${ }^{10}$ Lévy, Pierre: cit., p.12

${ }^{11}$ Pensemos que estos migrantes han recorrido un largo camino no sólo a través de geografías, sino también a través de tecnologías que posibilitan e imposibilitan sus desplazamientos, "la bestia" (tren de carga que recorre México hasta llegar a la frontera norte), la balsa, las divisas en diferentes países, "el pollero" (persona o personas a las cuales se les paga para ayudar en el cruce), las diferentes credenciales falsas e identidades que asumen en el recorrido para ocultar su lugar de origen, etc.

${ }^{12}$ Esta idea del Norte responde a una reconfiguración conceptual de la geografía mundial producto de la globalización, para la que en el primer mundo hay un dominio del trabajo inmaterial o cognitivo, desplazando el trabajo industrial al tercer mundo, propiciando que la principal fuente de plusvalía sean la producción y diseminación de signos, este proceso está acompañado lógicamente de la generación de sujetos deseantes de esos signos. "...lo global se materializa por fuerza en lugares específicos y acuerdos institucionales, gran parte de los cuales (si no todos) está localizada en territorios nacionales... desde el punto de vista internacional, las ciudades del norte global concentran más de la mitad del mercado global de capital." Sassen, Saskia: "La Ciudad Global: Introducción a un concepto", pp.51-53, tomado de http://www.cronicon.net/ForoUrbano/bogota/pdf/Documento1.pdf, consultada en abril 2015.

${ }_{13}$ Delgado, Manuel: "Seres de otro mundo: Sobre la función simbólica del inmigrante", p.15
} 


\section{CONCLUSIONES}

El registro estético en el que podemos ubicar a TBT, contemporáneo al fin, radica en la importancia que le da a todo lo contingente, su deliberada inoperatividad acusa ese aspecto, pero también lo hace la manera en que nos muestra a un cuerpo que está siendo afectado, nos lo muestra navegando; su identificación categorial "migrante ilegal" y la tecnología que lo informa, como indica Micha Cárdenas, colaboradora del proyecto, es una traducción en lo social y de lo social al arte que nos hace reflexionar sobre los intersticios de las estrategias artísticas. TBT apunta su larga ambición al objetivo de que el arte sea algo más que producción simbólica, dado que no sólo es esta la cualidad que puede insertarlo en lo social, busca hacer un levantamiento semántico que permita conceptualizar el siguiente paso. Este shifting model of affects (modelo de afectos cambiantes), como lo califica M. Cárdenas, nos habla de cuerpos que recusan ser afectos civilizados que actúen la felicidad del norte, por el contrario, comunican lo incivilizado, lo hostil, lo ajeno, lo indeseado. Visibilizar esos cuerpos, en el imaginario, en la imaginación, imaginar una narrativa de supervivencia que llega a un buen término, no es oponer un final feliz, es acusar lo contrario, es evidenciar los regímenes de poder, las sensibilidades que se han movilizado en torno al reforzamiento del límite. El proceso artístico de TBT es un punto de apoyo crítico para cuestionar la performatividad de la frontera, su extensibilidad al concepto Norte/Sur, y más importante aún, en lo que nos concierne, para mostrarnos con los lentes del poder, que se ha añadido estéticamente a estos cuerpos la cualidad de ser desechables. La desestabilización de ese registro tiene una traducibilidad social, tan la tiene que nos lleva a pensar, que esos cuerpos, sus cuerpos, lo son primero por el encuentro con otros cuerpos, encuentro que es el principio de la segregación sí, pero también de la afección y la alteridad, encuentro que es también de orden estético y que acusa una dinámica de proyección en lo real, una virtualización de la experiencia posible de hospitalidad y libre tránsito.

\section{FUENTES REFERENCIALES.}

BERARDI, Franco (Bifo). "The dark side of the multitud". En AA. VV., Impasse: dilemas políticos del presente. Colectivo Situaciones (Coord.) Buenos Aires: Tinta Limón, 2009. p.69-94.

BUTLER, Judith. El Género en Disputa. El feminismo y la subversión de la identidad. Muñoz, Ma. Antonia (Trad.) Madrid: Paidós, 2007. 318p. Studio; 168. ISBN: 9788449320309

Vulnerabilidad y Resistencia revisitadas, Conferencia, Sala Netzahualcóyotl, Ciudad Universitaria, UNAM, México D.F., 23 de marzo 2015.

CAMPILLO, Antonio, "Espacios de aparición: el concepto de lo político en Hannah Arendt". Daimon. 2002, Núm. 26, pp.159-186

CARDENAS, Micha. The Transborder Immigrant Tool, Science of the Oppressed. Antiatlas [en línea]. 2014 [ref. de 2014-05-07], disponible en: http://www.antiatlas.net/en/2014/05/07/conference-cardenas/ [Consulta 15 de abril 2015]

DELEUZE, Gilles. Diferencia y Repetición. Silva Delpy, María y Beccacece, Hugo (Trads.) Buenos Aires: Amorrortu Editores, 2002, 464p. ISBN: 950-518-361-5

DELGADO, Manuel. "Seres de otro mundo: Sobre la función simbólica del inmigrante". En La Dinámica del Contacto. Movilidad, encuentro y conflicto en las relaciones interculturales, II Training Seminar de jóvenes investigadores en Dinámicas Interculturales. Panel I. Espacios de/para la interculturalidad: Contacto, tránsito, apropiación [en línea]. Ministerio de Educación, Política Social y Deporte Español. Referencia SEJ 2006-12049 [Ref. de 04-2015]. Disponible en Web:

http://www.cidob.org/es/publicaciones/monografias/monografias/la_dinamica_del_contacto_movilidad_encuentro_y_conflicto_en_I as_relaciones_interculturales

Electronic Disturbance Theater [en línea], http://bang.transreal.org/ [consulta: abril 2015]

FOUCAULT, Michel. Defender la Sociedad. Curso en el Collège de France (1975-1976), François Ewald, Mauro Bertani y Alessandro Fontana en el marco de la Association pour le Centre Michel Foucault. México: FCE, 2001.

LEFEBVRE, Henri. The Production of Space. Nicholson - Smith, Donald (Trad.) UK: Blackwell Publishing, 1991. 434p. ISBN: 978-0-63118177-4.

LÉVY, Pierre: ¿Qué es lo virtual? Levis, Diego (trad.). Barcelona: Paidós, 1999. 126 p. ISBN: 84-493-0585-3

SASSEN, Saskia. La Ciudad Global: Introducción a un concepto [en línea], Cronicon [Bogotá, Colombia]: Foro Urbano, [ref. abril 2015] Disponible en Web: http://www.cronicon.net/ForoUrbano/bogota/pdf/Documento1.pdf 